

\section{Una Limpia en mi Honduras}

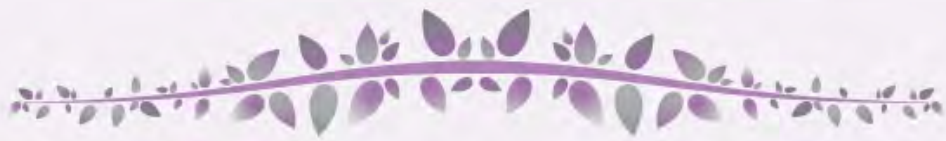

Voy a hacerle una fimpía a mi Honduras.

Voy a usar ruda, yerba buena, puros,

$$
\text { flor de izote, copal, guaro, }
$$

penicilina, agua limpia y justicia

Voy a invocar a las siete potencias africanas,

a Ixchel, diosa maya, a la Virgen de Suyapa

Voy a llamar a Yax Pak, a Lempira,

a Morazán, al Padre Subirana, a Visitación Padilla,

a Lorenzo Zelaya, a Manfredo Velasquez,

a Clementina Suárez, a los mártires y héroes

Voy a llamar al todo el pueblo Catracho.

Juntos haremos la limpia y sacaremos a los demonios

$$
\begin{aligned}
& \text { de la explotación, de la pobreza, } \\
& \text { del malinchismo, de la corrupción, } \\
& \text { del privilegio y la impunidad. } \\
& \text { Y limpios y libres } \\
& \text { encararemos al mundo del nuevo día. }
\end{aligned}
$$

Roberto Barahona

Poeta Hondureño 


\title{
Métodos Cuantitativos en la Búsqueda de Imaginarios Sociales. Estudio de Caso. Golpe de Estado del 28.06.09 en Honduras
}

\author{
FRANCESCA RANDAZZO EISEMANN ${ }^{1}$ \\ Recibido 10.05.16 / Aprobado 11.08.16
}

\begin{abstract}
RESUMEN. La finalidad de este trabajo es aproximarme a los imaginarios sociales del golpe de Estado del 28/06/09 en Honduras mediante la delimitación de un corpus textual de referencia, el establecimiento de unas marcas (Pintos, 2006) y el uso de programas informáticos. Se utiliza la teoría de los imaginarios sociales (Coca,Randazzo y Pintos, 2011) y el análisis léxico como técnica cuantitativa de estudio del discurso, complementado por codificaciones según los lineamientos de la Teoría fundamentada. En este trabajo se vislumbran una pluralidad de imaginarios que coexisten, algunos son comunes, otros diversos e inclusive antagónicos. Esto permite poner de manifiesto las fuerzas que promueven el cambio y aquellas que se oponen, así como las rupturas en los marcos y significados en los que se desenvuelve la sociedad hondureña en torno a esta crisis.
\end{abstract}

Palabras clave: Imaginarios sociales, golpe de Estado, léxico, Honduras.

ABSTRACT. The purpose of this work is to approach the Social Imaginary of the last coup d'état that took place in Honduras (June 6th, 2009), defining a textual reference, setting marks (Pintos, 2006) and using a specific software. Social Imaginary Theory (Coca et al., 2011) and Lexical Analysis as a quantitative technic are used to study the discourse, complemented with Grounded Theory. In this paper we show a plurality of Social Imaginaries that coexist -some are common, other are diverse and some are even antagonistic. This permits to reveal not only the forces that promote changes but also the opposite, as well as the rupture of frames and meanings in the Honduran society in this crisis context.

Keywords: Social Imaginaries, Coup d'état, lexicon, Honduras.

\section{Introducción}

La finalidad de este trabajo es aproximarme a una metodología de estudios de los imaginarios sociales a través de los discursos generados en torno al golpe de Estado del 28/06/09 en Honduras, temática ya desarrollada desde otras perspectivas en otros artículos varios (Randazzo, 2012a, 2013, 2015). En esta oportunidad se realiza explícitamente una delimitación de un corpus textual de referencia, el establecimiento de unas marcas ${ }^{2}$ y el uso de programas informáticos.

Para tener un panorama de la situación de Honduras entorno al golpe de Estado se plantea un conjunto de textos a partir de dos fuentes. La primera proviene de las compilaciones diarias de los titulares de los principales cuatro periódicos hondureños de mayor difusión recopilados por la Hemeroteca Nacional de Honduras.
La segunda procede de las compilaciones electrónicas enviadas por la Red de Desarrollo Sostenible.

Se han establecido unas marcas en base a la premisa según la cual todo lo que puede ser observado y descrito lo es por un observador con la ayuda de una distinción siguiendo la Teoría de sistemas. Pintos (2005) recuerda que lo que puede ser visto está determinado por el "lugar" desde donde se observa, complementando la afirmación por la consideración de la distinción Visibilidad/Invisibilidad, entendida desde lo que aparece «en el campo» y lo que queda «fuera de campo». Esto permitiría también lograr pensar lo que queda oculto y lo que se encuentra más allá de la polaridad, abriendo el espacio para esto-lo-otroaquello.

1. Doctora en Sociología, Universidad de Santiago de Compostela (USC), España (2012). DEA (máster) en Ciencias Políticas (Procesos políticos contemporáneos), USC, España (2010). Máster en Ciencias Sociales, Facultad Latinoamericana de Ciencias Sociales (FLACSO), Guatemala (2006). Licenciada en Lenguas (inglés), Universidad Autónoma, Honduras (1999). francescaensantiago@gmail.com.

2. "La observación sería una operación que utiliza una distinción para marcar una parte y no la otra. Una operación, por tanto, con dos componentes: la distinción y la indicación de la marca, que no pueden ser fusionadas ni separadas" (Pintos, 2004). 


\section{Metodología}

Se utiliza la teoría de los imaginarios sociales (Coca, 2011) y el análisis léxico como técnica cuantitativa de estudio del discurso, complementado por codificaciones según los lineamientos de la Teoría fundamentada. Se intenta poner de manifiesto las fuerzas que promueven el cambio y aquellas que se oponen, así como las rupturas en los marcos y significados en los que se desenvuelve la sociedad hondureña en torno a esta crisis.

Con el fin de acceder a una construcción de «la realidad» desde diversas perspectivas se hace recurso a una «observación de segundo orden», en la que se observa a un observador en cuanto observador, partiendo de un corpus de referencia, el cual es el locus de la evidencia interpretativa, para responder a las preguntas relevantes de la comunidad de interpretación en la que me inscribo según su propio lenguaje.

Se ve desde el lugar en el que cada cual se encuentra. Desde allí se tiene una perspectiva que privilegia ciertos aspectos, se desdeñan otros y no se perciben otros tantos más. Esto sucede en base al lugar especial que se ocupa con relación a la experiencia como un territorio intelectual e imaginario de producción de sentido. Una misma realidad será construida como diferente según se asuma una u otra perspectiva, dependiendo de la distinta ubicación social de los observadores.

\section{Uso de programas informáticos y teoría fundamentada}

El corpus fue codificado a través de dos programas informáticos Atlas.ti y MaxQDA ${ }^{3}$, que permiten trabajar con grandes cantidades de material textual. Estos programas han sido útiles en la creación de categorías de análisis para explorar e interpretar la información. Permiten realizar un "análisis continuo", es decir establecer una dialéctica entre el diseño y la revisión de la documentación existente, la producción de información y su análisis (Conde, 2009).

Con el fin de sistematizar el material y proceder a una codificación se ha utilizado la Teoría fundamentada (Grounded Theory), una de las técnicas clásicas utilizadas en ciencias sociales y que se encuentra en la base misma de la construcción y uso de las herramientas informáticas empleadas. Se trata de una metodología de análisis inductiva que propone teoría en base a datos empíricos. Mediante la comparación y aplicación sistemática de criterios de muestreo teórico abierto y saturación conceptual de las categorías encontradas, se estudian los procesos sociales a través de los datos (Charmaz, 2008). Las técnicas de la Teoría fundamentada prometen generar teoría creando conceptos provisionales que luego se van afinando, pero esencialmente permiten acercarse a aquello que subyace bajo la superficie.

El análisis se realiza a través de la creación de la unidad hermenéutica; asignación de los documentos primarios; generación de citas en los documentos primarios (primera reducción documental); codificación de los documentos primarios (segunda reducción documental); organización de las familias; establecimiento de las relaciones; creación de redes (networks); y confección de un texto final. Las categorías de análisis son en la medida de lo posible las mismas que utilizan los participantes de la investigación, conservándose así la materialidad y expresividad de las posiciones y expresiones (Charmaz, 2008).

En la lectura (lineal o por grupos centrales) se va creando un orden inteligible, provisional, el cual debe verse reflejado al menos en las anotaciones. Este es prácticamente un microanálisis, un examen minucioso de los datos y una primera interpretación de los mismos que incluye la codificación abierta y la axial. Durante el microanálisis se establecen hipótesis o enunciados sobre la forma en que se relacionan conceptos y categorías. Quedan así enfatizados los que se consideran los temas más significativos, así como las expresiones literales más significantes (Conde, 2009).

Los códigos ${ }^{4}$ marcan las direcciones analíticas para categorizar la información. A la hora de codificar, los temas van surgiendo a medida que se realizan las lecturas, siguiendo un estilo familiar en antropología. La codificación se puede realizar palabra por palabra, línea por línea, o incidente por incidente y se considera que hay varios tipos de códigos. Los códigos in vivo se toman de las frases que 'todos' usan y que condensan significado, capturan el sentido o la experiencia, o son particulares a cierto grupo. El código in vivo retoma del lenguaje de los actores. El código creado por el sociólogo es en cambio

3. Existen también otros tipos de QDA (qualitative data analysis) como Wmatrix (análisis y comparación, lista de frecuencias y concordancias, palabras clave y categorías gramaticales claves, dominios semánticos claves), NVivo (para textos de mucha riqueza o información multimedia que requiere profundos niveles de análisis), HyperResearch (una herramienta potente pero sencilla de análisis cualitativo), UCINET (análisis de datos de la red), ANACONDA (software de análisis cualitativo cuyas siglas significan Analyse de Contenu Disjoctive et Associative). Cabe destacar los trabajos del Semantic Technology Institute International (STII) considerado como el Think Tank líder en investigaciones sobre la semántica como pilar de la ingeniería informática.

4. Detrás de este proceso se puede ver la constitución de tipos ideales o de acción, objetivo principal de la sociología empírica de Max Weber. En este enfoque se tiende a construir tipos empíricos en el proceso de análisis orientado a un caso. Los tipos no tienen por qué ser necesariamente personas sino que también pueden ser tipos de acciones, situaciones, experiencias o actitudes. Se considera imprescindible asegurarse de que se conoce lo suficientemente bien el significado subjetivo de un texto, pues el teorema subyacente es el principio de "entendimiento interpretativo" basado en el concepto de interacción social de Weber. Cabe recalcar que el término "significado" para Weber comprende "cualquier tipo de significado verdadero objetivamente correcto o metafisicamente explorado". 
una construcción sociológica: los códigos descriptivos se refieren a aspectos narrativos de los sucesos; los códigos teóricos tienen una dimensión conceptual; los códigos sustantivos surgen directamente de los datos; la codificación axial establece relaciones en torno a una categoría tomada como eje.

\section{Contexto Teórico}

\subsection{El corpus}

Pintos (2005) propone observar los procedimientos por los que los diferentes emisores transmiten los rasgos definitorios de "su" realidad que pretende siempre ser "la" realidad. Para lograr esto, un observador de segundo orden debe determinar la distinción con la que los observadores de primer orden describen e interpretan: cómo y desde dónde observan, cómo seleccionan lo que perciben, cómo utiliza los sentimientos, las emociones, la cercanía o la lejanía objetivante, etc.

El corpus está conformado por las compilaciones diarias de los titulares de los principales cuatro periódicos hondureños de mayor difusión (El Tiempo, El Heraldo, La Prensa y La Tribuna) recopilados por la Hemeroteca Nacional de Honduras (en adelante llamada HNH) y enviados por subscripción electrónica regularmente hasta el momento del golpe. La segunda procede de las compilaciones electrónicas enviadas por la Red de Desarrollo Sostenible (en adelante llamada RDS), en la que se agrupan las aportaciones de diversos ciudadanos sobre temáticas que tocan de una manera u otra la situación política y social de Honduras, sobre todo en la medida que la fecha del golpe se acerca ${ }^{5}$. Mientras los envíos de la $\mathrm{HNH}$ eran el lugar electrónico idóneo para informase, a partir del 28/06/09 su recepción no es regular y RDS es una fuente alternativa de información desde adentro ${ }^{6}$.

\subsection{Las marcas}

Mediante el establecimiento de unas marcas se trata de englobar múltiples puntos de vista, para tener un efecto caleidoscópico de la sociedad. El corpus conforma la fuente de «observación de primer orden». Puestas las marcas, se trata de explotar la posibilidad de la distinción adecuadamente, sacar partido de la escisión a través del manejo de los programas informáticos. Según la metodología de estudio de los imaginarios sociales de Pintos (2001, 2005), además de elaborar una distinción e indicación de la marca, habría que establecer el modo y la manera cómo se observa, marcar que se está de un lado y no de otro. En esta perspectiva, el observador es lo noobservable.

Según quién sea el observador, un mismo fenómeno será visto de distintas maneras. Lo relevante para algunos, no lo será para otros, pero estará indicando lo que ve y no ve el observador. Las personas con diferentes visiones de mundo piensan de forma distinta y pueden llegar a conclusiones diferentes a partir de los mismos hechos aunque el racionalismo postule la razón universal. Lakoff y el Rockridge Institute (s.f.) sostienen que es por eso que para encontrar los marcos o esquemas mentales es necesario repensar el racionalismo (el cual dice que es la razón la que nos hace humanos e igualmente razonables) así como el iluminismo y los fundamentos del sistema democrático.

Interpretar de una manera las cosas significa también dejar de hacerlo de otra, así como ver hacia un lado implica no ver hacia el otro: porque no vemos es que vemos. Pintos (2001) considera que precisamente los imaginarios serían lo que vemos de lo que no vemos. Al observar un mismo fenómeno, cada cual se ubica de un lado de la realidad social. Desde allí describe "el otro lado", ubicándose en un lado en el que no se es consciente de lo que no sabe, y quizá tampoco de lo que se sabe. Esto queda claro cuando se intenta hacer explícitos los postulados con los cuales se construye los razonamientos. No es imposible, pero tampoco evidente.

Conde (2009) lo llama el lugar social, el cual puede configurarse en función de dimensiones clásicas como sexo, edad, profesión, hábitat, sentirse representante de... Los usuarios de la lengua realizan su construcción subjetiva según las posiciones que ocupan como participantes (edad, género, poder, etc.), pero "no es el hecho objetivo de que los hablantes sean hombres o mujeres, jóvenes o viejos, poderosos o no, sino cómo se ven y se construyen a sí mismos, en general o en la situación social en desarrollo" (Van Dijk, 1998). Para Pintos (2005) los siguientes elementos han de ser tomados en cuenta: rural, urbano, suburbano, encrucijada, frontera, participante, no participante, visión emic o etic, creencias religiosas y políticas, roles diferenciados en el trabajo, familia, vida cotidiana, sexo, clase, etc.

Usando el símil de la cámara fotográfica o de la papila óptica del ojo, desde cada perspectiva existe un "punto ciego", que impide ver y permite ver (Pintos, 2005). En

RDS brinda acceso también a otro tipo de informaciones, tales como un boletín de empleos, eventos, noticias culturales, foros virtuales, etc. Ver http://rds.hn/

6 Aunque no se incluya en este estudio, especial atención debe darse también al rol de las redes sociales como Facebook en este contexto político, pues jugaron un rol determinante en la difusión alternativa e inmediata de la información, sobre todo durante los toques de queda que fueron prácticamente diarios alrededor de varias fechas álgidas. 
el ámbito neurocognitivo, los puntos ciegos se encuentran en los ojos (en posiciones ligeramente diferentes en cada ojo) y es el uso de ambos ojos, junto con la interpretación del cerebro, la que no nos permite percatarnos de él. Sin embargo, y aunque el cerebro siga intentando impedírnoslo, si cerramos o tapamos un ojo podemos llegar a detectar dicho punto ciego. En el sentido de los imaginarios sociales, se trataría de remarcar distintas observaciones, en las cuales quede de manifiesto el punto ciego, como al mirar con un solo ojo.

Frente a la noción de "punto ciego", cabe mencionar que otros autores han hablado de "lugares privilegiados" para observar la realidad. No se puede ver todo desde todas partes, esto implica también que hay partes desde las que se puede "ver", "entender", construir realidad con una determinada perspectiva, quizá privilegiada. Ciertas cosas no pueden ser vistas, pasan desapercibidas si no se posee cierta base, o si no se han desarrollado ciertas cualidades o capacidades para poder verlas.

Esto señala hacia los intereses, las motivaciones profundas. Ciertamente el ángulo de observación determina lo observable también y hay todo un universo que al seleccionar queda fuera de foco. Ya no se trata sólo de no poder ver, sino de decidir no tomar ciertas cosas en cuenta, como al enfocar y dejar fuera de foco. Para ver y comprender lo que ocurre en una sociedad de clases es indispensable ocupar ciertas posiciones y no otras. Para poder escapar a la lógica de la ideología dominante, para percibir la esencia de una realidad necesariamente conflictual hay que ocupar determinadas posiciones en el conflicto, como propone el marxismo.

Cómo se selecciona lo que puede ser visto, no es sólo es un acto de libertad sino que se encuentra tanto delimitado como potenciado por un imaginario social, unos marcos mentales que al ser utilizados determinan la forma de dar sentido. Para Pintos (2005) la función primaria de los imaginarios sociales es la elaboración y distribución generalizada de instrumentos de percepción de la realidad social construida como realmente existente. Este umbral de visibilidad-que implícitamente niega o permite diferentes posibilidades de lo existente o de lo posible - se encuentra influenciado por la institucionalización de un magma de particulares significaciones que dotan de un sentido a las cosas. Las significaciones imaginarias consiguen que una determinada percepción de lo real adquiera el rango de evidencia (Castoriadis, 1975).

\section{- Primera marca: la fuente}

La fuente, está dispuesta para diferenciar dos emisores -la Hemeroteca Nacional de Honduras (HNH) y la Red de Desarrollo Sostenible (RDS)- y comparar sus discursos.

En principio, son dos posiciones distintas: la de los medios escritos (periódicos) cuyos enfoques y escritos están contralados; y la de un medio electrónico abierto a cada quien que desee participar (todo lo enviado es publicado).

La primera fuente tiene un alto grado de legitimidad; la otra en cambio, es un espacio alternativo, desde el punto de vista de Williams (1980) se trataría de una "preformación". Los sesgos de RDS y HNH, responden también a ciertos criterios. Aquí hay que tener en cuenta que tal interés es diferente de la suma de los intereses particulares (de quienes envían información a RDS o los periódicos de los que se sustraen los titulares la Hemeroteca) y responde a una primera "manipulación" de selección/orden/titulación.

\section{- Segunda marca: el tiempo}

Los textos que conforman la fuente de «observación de primer orden» fueron producidos un mes antes y una semana después del golpe de Estado del 28 de junio de 2009. Tanto en el caso de las compilaciones de la $\mathrm{HNH}$ como de la RDS se analizan dos bloques: entre el 27 de mayo y el 27 de junio de 2009 y entre el 28 de junio y el 5 de julio de 2009. El conjunto de textos está enmarcado entre la fecha del terremoto del 28 de mayo (en Honduras muy raramente son perceptibles los movimientos de placas) y el 5 de julio, día del primer intento fallido de Zelaya por regresar a Honduras, eventos que a priori marcan un giro en el curso del devenir social.

En total son 138 emisiones informativas. Los días en que más emisiones hay son (fecha y a continuación número de emisiones): 04-06-09, 6; 10-06-09, 8; 12-06-09, 7; 17 06-09, 6; 23-06-09, 7; 25-06-09, 5;26-06-09, 7; 27-06-09, 7 ; 30-06-09, 5; 01-07-09, 8; 03-07-09, 7; 04-07-09, 9; 0507-09, 10.

\section{- Tercera marca: periódicos y tipos de lista}

RDS envía diversas "listas" de correos: Clasificados (1), Becasycursos (1), Empleos (6), Eventos (2), libertadexpresion ${ }^{7}$ (66), arteycultura (36). Además, he

7. "Libertad de Expresión" -la lista que recopila principalmente la información aquí utilizada- se define en la web como "un espacio de opinión pública, cada mensaje representa la opinión exclusiva de la persona que lo escribe, está respaldado con el nombre de usuario registrado en el Portal el cual tiene un correo electrónico válido para su contacto". 
trabajado con el tipo de texto (por mí determinado) que conforma los envíos de RDS: Convocatoria (13), Pronunciamiento/Comunicado (58), Documento legal (19), Enlaces internet (99), Narración informativa (71), Narración estilo periodístico (127), Narración estilo comentario (203), Opinión (16), Ficción (3), Poema (16). En cuanto a la $\mathrm{HNH}$, los diversos periódicos son $\mathrm{El}$ Tiempo, El Heraldo, La Prensa y La Tribuna, y contienen diversas secciones que incluyen los de titulares de las noticias: Nacionales, Culturales, Económicas, Políticas, Internacionales, Editoriales, Deportivas (aunque de los 26 envíos sólo en uno se incluye una sección deportiva). Sus envíos contienen diversa cantidad de titulares según el periódico: La Tribuna (227), El Heraldo (261), La Prensa (212), El Tiempo (194).

\section{- Cuarta marca: emisores diferenciables en ambas fuentes}

Otra forma de establecer una marca es según el sujeto específico que habla. La mayor parte de los envíos están escritos por la persona que los remite (324), pero una buena cantidad envía textos que proceden de otra fuente (216). Los textos en su mayoría están escritos en español, pero un pequeño porcentaje está en inglés y en italiano.

Los mensajes provienen de 56 distintas organizaciones de la sociedad civil, 397 personas con nombre y apellido (96 mujeres, que representan un $23,82 \%$ ), 27 personas con mote (RDS) y 51 remitentes anónimos. De estas últimas 29 personas intervienen más que las otras (6 mujeres, las cuales representan un $20,68 \%$ ).

\section{Discusión de resultados}

Por cuestiones de espacio y la necesidad de explorar exclusivamente los aspectos cuantitativos que fueron explorados, se reportan sólo algunos de los hallazgos y no su totalidad. Estos están referidos a la manera en que se clasificó el corpus, la codificación y el análisis léxico.

\subsection{Corpus}

El corpus se encuentra limitado por los textos generados del 27 de mayo al 5 de julio ${ }^{8}$. Entre el 27 de mayo y el 27 de junio de 2009 se efectúan 66 envíos de RDS (de los cuales 26 tratan sobre el acontecer político), y 23 envíos de la Hemeroteca (más 4 envíos más llamados "Esta semana en la historia"); entre el 28 de junio y el 5 de julio de 2009 se hacen 46 envíos RDS y 3 de $\mathrm{HNH}^{9}$ (más uno de "Esta semana en la historia"). Como se puede ver, proporcionalmente, los mensajes de RDS casi se triplican a partir del golpe, mientras que los de $\mathrm{HNH}$ disminuyen prácticamente a la mitad. También es necesario notar que en RDS, a partir del golpe, todos los envíos tratan exclusivamente sobre la situación política. En total, se trata de un corpus que contiene 284,329 palabras en unas 710 páginas aproximadamente.

El corpus se considera como un diálogo, pues tienen continuidad en el tiempo, aunque no en el espacio. Por lo tanto, la unidad de análisis es el corpus de textos visto como una totalidad, un espacio de intercambios entre discursos, los cuales contienen una carga simbólica que sitúa a la comunidad hondureña en un tiempo determinado en el que se relaciona con su pasado, su entorno y con el poder. A continuación los textos estudiados (los títulos están formados por la fecha de envío y el nombre del correo electrónico), dispuestos en orden descendente con relación a la fecha de envío:

\section{RDS 28/06-05/07}

05-07-09 Más envíos sobre los sucesos del 5 de julio 05-07-09 Hoy una familia llora la muerte de un muchacho 05-07-09 Día 5 de julio termina la manifesta 05-07-09 Honduras 5 de julio 2009 sexto envío de mensajes 05-07-09 Séptimo día 5 de julio quinto envío 05-07-09 Honduras 5 de julio 2009 cuarto envío de mensajes 05-07-09 Séptimo día tercer envío de mensajes 05-07-09 Séptimo día 5 de julio segunda entrega 05-07-09 Séptimo día 5 de julio 2009

05-07-09 Séptimo día de resistencia Honduras

04-07-09 Teatreros salvadoreños en frontera honduro

04-07-09 Boletín de empleos 4 de julio

04-07-09 Resistencia al Golpe de Estado

04-07-09 Antesala al eminente retorno de Zelaya

04-07-09 Expresiones particulares sobre el golpe

04-07-09 Expectativas del retorno de Zelaya

04-07-09 Secretario de OEA visita Honduras

04-07-09 Una Perspectiva diferente

04-07-09 Denuncian represión similar a los años ochenta

03-07-09 Organismo de Derechos Humanos

03-07-09 E1 hoy de la resistencia

03-07-09 El poder de la libertad

03-07-09 Denuncias de Represión

03-07-09 Libertad

03-07-09 Comunidad internacional continúa desaprobando golpe

02-07-09 Mensaje enviado 2de julio quinta parte

02-07-09 Mensajes 2 de julio segunda parte

02-07-09 Envío de mensajes 2 de julio

01-07-09 Congreso suspende garantías constitucionales en Honduras

01-07-09 Resistencia Popular Continua

8. El total del material utilizado, así como las codificaciones informáticas se encuentra en versión electrónica y a la disposición para futuras investigaciones.

9. De 138 emisiones informativas, 112 corresponden a RDS y 26 a HNH. Tanto la totalidad de los textos como el trabajo de codificación realizado tiene un potencial de análisis que sobrepasa las capacidades de la tesis doctoral de la cual se desprende este artículo, pero que puede ser explotado en un futuro. 
01-07-09 Segunda parte

01-07-09 Persistencia por la Democracia parte 2

01-07-09 Mensaje primero de julio

01-07-09 Defendamos la Constitución!

01-07-09 12 mensajes del 20 de junio en este envío

30-06-09 Denuncia ante el Ministerio Público

30-06-09 Continúan las acciones de protesta

30-06-09 Marcha por la paz

30-06-09 Honduras en Rebeldía

30-06-09 Actividad Cultural Pospuesta

29-06-09 Persistencia por la Democracia

29-06-09 Secuestros de dirigentes de organizaciones sociales

28-06-09 Honduras 28 de junio $9 \mathrm{p}$

28-06-09 Honduras 28 de junio 5 p

28-06-09 Reportan muerte de líder popular al resistir al arrest

28-06-09 Urgente

\section{Hemeroteca 28/06-05/07}

03-07-09 La Hemeroteca le Informa

02-07-09 La Hemeroteca le Informa

01-07-09 La Hemeroteca le Informa

\section{RDS $28 / 05-27 / 06$}

27-06-09 RE Posición IV Uma, RDS

27-06-09 porque, RDS

27-06-09 Honduras es Primero, RDS

27-06-09 Fw Mel y Su Grupo de Inmorales y Comuptos, RDS

27-06-09 Con la vara que midas, serás

27-06-09 siete mensajes en este envío, RDS

26-06-09 SRC puros

26-06-09 invitación

26-06-09 Galería fotográfica a su disposición

26-06-09 seis mensajes en este envío, RDS

26-06-09 La hora de la razón, RDS

26-06-09 siete mensajes en este envío, RDS

25-06-09 La derecha quiere un 11 de abril

25-06-09 Dos mensajes en este envío

25-06-09 Posición IV Uma

25-06-09 Documentos de Eduardo Barh

24-06-09 Comunicado COPA

24-06-09 V CONCIERTO DE TEMPORADA EN SPS

24-06-09 Boletín de empleos 24 de junio

23-06-09 CONCIERTO DE GALA Las Últimas Sinfonías

23-06-09 Presentación de libros Corral de locos

23-06-09 LA POSADA DE MIRANDOLINA de Goldoni

23-06-09 Presentación de libros Corral de locos

23-06-09 Cuatro mensajes en este envío

23-06-09 Exposición fotográfica de la Cruz Roja en la Alianza F

22-06-09 Invitación al estreno de nueva producción teatral

22-06-09 Este 23 y 25 de junio Café Paradiso

19-06-09 Unete al Plantón De Este Viernes

18-06-09 Paso la Bola, el gobierno de Mel y sus opositores

17-06-09 PROMOCION DE FERIA JUNIANA

17-06-09 Paso La Bola

17-06-09 Invitación al II Aniversario del Centro Documental

17-06-09 Muestra colectiva de fotografía Cuidad Imaginada

17-06-09 Boletín de empleos 17 de junio

16-06-09 Conferencia tema Encuesta de Opinión

16-06-09 TALLER DE TEATRO AEREO

16-06-09 Boletín de Clasificados 16 de junio

15-06-09 CAMBIO DE FECHA PARA EVENTO
15-06-09 Café Paradiso Visita Blog

15-06-09 Boletín de Becas y Cursos 15 de junio

12-06-09 Hoy 12 de junio Café Paradiso presenta lectura de

12-06-09 Anexo empleos 12 de junio

12-06-09 Gran Tele

12-06-09 sonando en Rock en Rebelión

12-06-09 Sebastiana

11-06-09 E1 Inventario, blog contra el cinismo y la entrega, RD

11-06-09 DESDE LA MOVILIZACION DE LA IV URNA

11-06-09 Sito web Hondubirding and Nature Blog

10-06-09 Boletín de empleos 10 de junio

10-06-09 Casting para cine

10-06-09 Fiesta de la Música 2009

10-06-09 Noche beneficiosa Pro

10-06-09 EVENTO HISTORICO

10-06-09 Revista de críticas y respuestas, RDS

10-06-09 BALLET CLÁSICO DE MOSCÚ EN HONDURAS

08-06-09 Cine centroamericano Café Paradiso

08-06-09 Boletín de Becas y Cursos 8 de junio

08-06-09 Invitación de MUJERES SOCIALISTAS-LOLAS, RDS

05-06-09 Evento de HIP HOP

04-06-09 Vargas Llosa tiene un lucero en la frente y tinieblas

04-06-09 V Jornada de Bibliotecología en Honduras

04-06-09 ALBA Cultural, RDS

04-06-09 Presentación artística del CCAA

04-06-09 II Segunda Feria Ambiental

03-06-09 Boletín de empleos 3 de junio

\section{Hemeroteca 28/05-27/06}

27-06-09 La Hemeroteca le Informa

26-06-09 La Hemeroteca le Informa

25-06-09 La Hemeroteca le Informa

24-06-09 La Hemeroteca le Informa

23-06-09 La Hemeroteca le Informa

19-06-09 La Hemeroteca le Informa

18-06-09 La Hemeroteca le Informa

17-06-09 La Hemeroteca le Informa

16-06-09 La Hemeroteca le Informa

13-06-09 La Hemeroteca le Informa

12-06-09 La Hemeroteca le Informa

11-06-09 La Hemeroteca le Informa

10-06-09 La Hemeroteca le Informa

09-06-09 La Hemeroteca le Informa

08-06-09 La Hemeroteca le Informa

04-06-09 La Hemeroteca le Informa

03-06-09 La Hemeroteca le Informa

02-06-09 La Hemeroteca le Informa

01-06-09 La Hemeroteca le Informa

30-05-09 La Hemeroteca le Informa

29-05-09 La Hemeroteca informa

28-05-09 La Hemeroteca le informa

27-05-09 La Hemeroteca le informa

\subsection{Codificación}

Cabe precisar que Atlas.ti fue utilizado sólo en ciertos textos, y que el sistema de códigos más grande fue realizado en MaxQDA. Éste se transcribe a continuación: 


\section{Clima sociopolítico}

Caudillismo

Continuismo

Asamblea Nacional Constituyente

Constitución

Diálogo

Cuestionamiento

Perspectivas

Debate

Inestabilidad

Cambio

Idealismo

Solución conflicto

Democracia, institucionalidad

Derechas

Izquierdas

Terrorismo

Derechos hum, constituc.

Obediencia

Violencia

Paz

Duda, división

Conflicto

Estado de excepción

Medidas cautelares

Tomas de carreteras, edificios

Liderazgo

Electoral/ partidos

Sanciones

Legitimidad

Cuarta Urna

Toque de queda

Judicial/justicia

Golpe

Legal

Crisis social y política

Crisis política

Política/escenario internacional

Políticas interna

Otros temas

Muerte

Libertades

La lucha

Lo histórico

Regreso/retroceso/repetición/restitución

Razones y responsables

Análisis

Consecuencias económicas

Alimentación

Vivienda

Salario

Interés en Honduras

Agro

ALBA

Armas

Artes

Bloqueo económico

Centroamérica

Clima

Combustible

Crisis financiera

Cuba
Vivienda

Salario

Interés en Honduras

Agro

ALBA

Armas

Artes

Bloqueo económico

Centroamérica

Clima

Combustible

Crisis financiera

Cuba

Deuda

Diplomacia

Ecología

Educación

Embates del clima

Empleo/Laboral

Encuesta/ consulta

Energía eléctrica

Finanzas

FMI

Fútbol

Guerra

Homosexualidad

Impuestos

Indemnizaciones

Infraestructura

Investigación

Maquilas

Mercado

Migración

Narcotráfico

Nota roja/ amarillismo

Pacto político

Pobreza

Presupuesto

Recurso forestal

Registro

Remesas

Salud

Servicios

Sismo

Telecomunicaciones

Transportes

Turismo

Venezuela

Estados emocionales, sentimientos

El Pueblo

USA

Empresarios

Escritores

Estudiantes

Excluidos

Fuerzas Armadas

Gobierno

Iglesias/religión

Indígenas y etnicidad

Insulza

Intelectuales

Jóvenes 
Deuda

Diplomacia

Ecología

Educación

Embates del clima

Empleo/Laboral

Encuesta/ consulta

Energía eléctrica

Finanzas

FMI

Fútbol

Guerra

Homosexualidad

Impuestos

Indemnizaciones

Infraestructura

Investigación

Maquilas

Mercado

Migración

Narcotráfico

Nota roja/ amarillismo

Pacto político

Pobreza

Presupuesto

Recurso forestal

Registro

Remesas

Salud

Servicios

Sismo

Telecomunicaciones

Transportes

Turismo

Venezuela

Estados emocionales, sentimientos

Alegría

Tristeza

Desafiante

Vergüenza

Optimista

Dolor

Confianza

Solidaridad

Desconfianza

Solidaridad (1)

Esperanza

Ingenuidad

Alerta

Repudio

Indignación

Confusión, incredulidad, shock

Temerarios

Indefensión (1)

Desafiantes

Ironía

Miedo

Relación

Desobediencia civil

Polarización/antagonismo

Represión

Participación

Persecución
Magisterio

Maras

Medios/información

Morazán

Movimiento popular/ sociales

Mujeres

Niñez

OEA

ONGs

ONU

Políticos

Arias

Chávez

Correa

Elvin Santos

Fúnez

Hillary Clinton

Micheletti

Pepe Lobo

Zelaya

Prensa

Secretaría de Cultura

Sindicatos

Soc Civil

Universidad

Tipo de texto

Ficción

Convocatoria

Documento legal

Enlaces internet

Narración estilo comentario

Narración informativa

Narración estilo periodístico

Opinión

Poema

Pronunciamiento; Comunicado

Secciones medios

Nacionales

Culturales

Económicas

Políticas

Internacionales

Editoriales

Arte Acción

Asociación de Est. de Sociología

Asociación de Jueces AJD

Asociación Filarmónica Coral

Asociación Tiempos Nuevos

Teatro

Attac Chile

Boletín Informativo "El Catracho"

C-libre

Centro Cultural Arte y Amistad

Centro Cultural Infantil

CODDEFFAGOLF

Codeh

COFADEH

COMHTE

COMPA

Comunicación Comunitaria

$\mathrm{CONIMCHH}$

COPINH

CPTRT 
Dominación

Enfrentamiento

Indefensión

Denuncia

Condena, rechazo, repudio

Movilización, manifestaciones

Confrontación

Resistencia

Silenciamiento

Revolución/ rebelión

Corrupción

Insurrección

Organización popular

Identidades/ Actores diferenciables

Frente de resistencia popular

Depuestos

Golpistas

Europa

Involucrados, responsables

Alcaldía

Patria

Artistas

Cafetaleros

Campesinado

Policía

Género

Clase media

Congreso

Cooperación internacional

El Pueblo

USA

Empresarios

Escritores

Estudiantes

Excluidos

Fuerzas Armadas

Gobierno

Iglesias/religión

Indígenas y etnicidad

Insulza

Intelectuales

Jóvenes

Magisterio

Maras

Medios/información

Morazán

Movimiento popular/ sociales

Mujeres

Niñez

OEA

ONGs

ONU

Políticos

Arias

Chávez

Correa

Elvin Santos

Fúnez

Hillary Clinton

Micheletti

Pepe Lobo

Zelaya

Prensa

Secretaría de Cultura
Editorial

El Demócrata

Embajada de Francia

Espacio Regional de Occidente

Estudiantes de Posgrado Educación

Festival del Caribe

FIAN

Frente contra el Golpe

Frente Nacional contra el Golpe

Fundación Puntos de Encuentro

Guaymuras

Habla Honduras

Honduras Educacional

IHAH

Instituto Juan Manuel Gálvez

La Vía Campesina Internacional

Lenca Land Trials

MASTA

Moskitia Asla

Movimiento Amplio por la

Dignidad

Movimiento Mujeres Soc.

Las Lolas

OFRANEH

Organización política Los Necios

Persistencia Democrática

PINU Cortés

Popol Nah Tun

Proyecto Gritón

Radio Progreso

RDS

Secretaría de cultura

Sociedad Civil Santa Rosa de

Copán

Transparencia Red de Occ.

Emisores particulares y anónimos 
Sindicatos

Soc Civil

Universidad

Tipo de texto

Ficción

Convocatoria

Documento legal

Enlaces internet

Narración estilo comentario

Narración informativa

Narración estilo periodístico

Opinión

Poema

Pronunciamiento; Comunicado

Secciones medios

Nacionales

Culturales

Económicas

Políticas

Internacionales

Editoriales

Deportivas

Fechas

27-05-09

28-05-09

29-05-09

30-05-09

01-06-09

02-06-09

03-06-09

04-06-09

05-06-09

08-06-09

09-06-09

10-06-09

11-06-09

12-06-09

13-06-09

15-06-09

16-06-09

17-06-09

18-06-09

19-06-09

22-06-09

23-06-09

24-06-09

25-06-09

26-06-09

27-06-09

28-06-09

29-06-09

30-06-09

01-07-09

02-07-09

03-07-09

04-07-09

05-07-09
Emisores medios (periódicos)
La Tribuna
El Heraldo
La Prensa
El Tiempo

Listas RDS

Clasificados

Becasycursos

Empleos mailing list

Eventos

libertadexpresion

arteycultura

Emisores organizaciones

COPA

Adital-noticias

Agencia Prensa Latina

ANDEH

ANMCLA Caracas

\subsection{Tokens y verbatims}

Con MaxQDA se analizó todo el material generando segmentos temáticos que incluyen la localización exacta dentro del texto y conforman, junto a sus respectivas identificaciones. A continuación, como ejemplo, uno de los segmentos codificados en MaxQDA:

\section{Texto: $\quad$ Hemeroteca 28/06-05/07\03-07-09 La Hemeroteca le Informa \\ Peso: $\quad 100$ \\ Posición: $3-3$ \\ Código: Clima sociopolítico\Golpe \\ “Congreso perpetró un golpe, asegura Edmundo Orellana”}

A partir de una lectura cuidadosa se seleccionan fragmentos de texto para posteriormente efectuar los análisis oportunos. En el momento del análisis de los segmentos codificados, se deben buscar los verbatims más adecuados para ilustrar las líneas argumentales, es decir, aquellos condensan la totalidad (Conde, 2009), lo cual queda a consideración de los criterios del investigador.

En la investigación efectuada, la mayor cantidad de tokens corresponden a las Fuerzas Armadas, los medios de comunicación, Zelaya y Micheletti (Cuadro No 3 ) y hay una serie de participantes de muy diversas categorías entre ellos actores clásicos: Alcaldía, Policía, Congreso, Cooperación internacional, Fuerzas Armadas, Gobierno, Iglesias, Medios, Clase media, Prensa, Secretaría de Cultura, Sindicatos, Sociedad Civil, Universidad, Movimiento popular, Movimientos sociales, Frente de resistencia 
popular; identidades: Artistas, Cafetaleros, Campesinado, Empresarios, Escritores, Estudiantes, Grupos étnicos, Intelectuales, Jóvenes, Magisterio, Niñez, Mujeres, Políticos, Maras; individuos: Insulza, Morazán, Arias, Chávez, Correa, Elvin Santos, Fúnez, Hillary Clinton, Micheletti, Pepe Lobo, Zelaya; organizaciones: OEA, ONGs, ONU; países: Europa, USA; nuevas identidades: Depuestos, Golpistas, Involucrados, Responsables, Excluidos, El Pueblo, La Patria.

\subsection{Estudio léxico}

Una vez que las opiniones se vuelven convencionales, se codifican en el léxico, siendo éste por lo tanto un componente indiscutible del análisis del discurso. "La forma más obvia, y por lo tanto más ampliamente estudiada, de expresión ideológica en el discurso puede encontrarse en las palabras escogidas para expresar un concepto" (van Dijk, 1998).

$\mathrm{Al}$ prestar atención al léxico de todo el corpus, está claro que si bien Zelaya es la chispa que enciende la llama, el eje central de las noticias y entradas referentes al ámbito político (tanto en RDS y $\mathrm{HNH}$ ) es la confrontación. La oposición está expresada en el vocablo predominante "no". La segunda palabra más utilizada es "Honduras" ubicándole en el tema de la disputa (Cuadros $\mathrm{N}^{\circ} 1$ y 2).

Cuadro $\mathrm{N}^{0} 1$. Palabras más usadas diferenciadas por la fuente un mes antes (27/05 a $27 / 06)$

\begin{tabular}{|l|l|}
\hline \multicolumn{2}{|c|}{ Un mes antes del golpe } \\
\hline RDS & HNH \\
No Honduras & No \\
desarrollo & junio \\
experiencia & cuarta urna \\
proyecto & poder \\
trabajo & ejecutivo \\
junio & Honduras \\
pueblo & crisis \\
proyectos & Zelaya \\
presidente & gobierno \\
urna & democracia \\
democracia & constitución \\
Zelaya & influenza \\
constitución & encuesta \\
consulta & presidente \\
encuesta & asamblea \\
justicia & gripe \\
\hline
\end{tabular}

Un mes antes, el tema del golpe y la cuarta urna están presente sobre todo en $\mathrm{HNH}$ cuyo segundo énfasis es la influenza o gripe A, mientras que RDS se enfoca más hacia lo que le es propio, es decir el trabajo, los proyectos, aunque también se encuentran presentes los mismos temas que en $\mathrm{HNH}$. Una semana después -a pesar de algunas diferencias que se resaltan-se iguala el tema predominante (no, Honduras, Zelaya), y surge en $\mathrm{HNH}$ la referencia al diálogo y la paz (Cuadros $\mathrm{N}^{\circ} 1$ y 2).

Cuadro $N^{0}$ 2. Palabras más usadas diferenciadas por la fuente una semana después $(28 / 06-05 / 07)$

\begin{tabular}{|l|l|}
\hline \multicolumn{2}{|c|}{ Una semana después del golpe } \\
\hline RDS & HNH \\
No & No \\
Honduras & diálogo \\
presidente & Zelaya \\
Zelaya & Mel \\
Estado & paz \\
pueblo & Micheletti \\
golpe & golpe \\
poder & Honduras \\
\hline
\end{tabular}

Las palabras más usadas en todo el corpus refieren a la Colectividad/comunidad: Honduras, País, Pueblo, Todos; a la Política: Presidente, Zelaya, Gobierno, Poder, Democracia, Golpe; y a las Leyes: Constitución, Derechos. Aunque Zelaya como palabra no es la más utilizada (sexto lugar), se puede considerar que como tópico es el más frecuentes, al tener la mayor cantidad de tokens (307).

Cuadro $\mathrm{N}^{\circ} 3$. Palabras comunes y prioridad, diferenciadas por la fuente $(27 / 05$ a $27 / 06)$

\begin{tabular}{|l|l|}
\hline HNH & RDS \\
NO & NO \\
HONDURAS & HONDURAS \\
CUARTA URNA & CUARTA URNA \\
GOBIERNO & ZELAYA \\
ZELAYA & GOBIERNO \\
\hline
\end{tabular}

Cuadro $\mathrm{N}^{\circ}$ 4. Palabras más usadas diferenciadas por la fuente (una semana después)

\begin{tabular}{|l|l|}
\hline RDS & HNH \\
Honduras & diálogo \\
presidente & Zelaya \\
Zelaya & Mel \\
Estado & paz \\
pueblo & Micheletti \\
golpe & golpe \\
poder & Honduras \\
\hline
\end{tabular}


Destaca el personalismo: Micheletti, Elvin, Zelaya, Mel; el Estado de Derecho: plebiscito, elecciones, constitución, democracia, ley, presidente, cuarta urna; la relación de fuerzas: política, gobierno, poder, golpe, crisis; las instituciones (para Williams (1976), cualquier elemento organizado de la sociedad): secretaría, MDC, TSE, asamblea, congreso.

Cuadro $\mathrm{N}^{\circ} 5$. Comparación de las palabras más usadas tanto en HNH como RDS (27/05 a 27/06)

\begin{tabular}{|l|c|c|l|c|c|c|}
\hline \multicolumn{1}{|c|}{ HNH } & Cantidad & Porcentaje & \multicolumn{1}{c|}{ RDS } & Cantidad & Porcentaje \\
\hline NO & 85 & $\mathbf{2 , 7 2}$ & NO & 390 & 6,35 \\
\hline HONDURAS & 70 & $\mathbf{2 , 2 4}$ & PUEBLO & 183 & 2,98 \\
\hline CUARTA & $\mathbf{5 6}$ & 1,79 & SI & 122 & 1,99 \\
\hline CULTURA & $\mathbf{5 5}$ & 1,76 & HONDURAS & 118 & 1,92 \\
\hline POLÍTICA & 45 & 1,44 & PRESIDENTE & 118 & 1,92 \\
\hline PRENSA & 37 & 1,18 & ESTADO & 97 & 1,58 \\
\hline GOBIERNO & 36 & 1,15 & URNA & 93 & 1,51 \\
\hline URNA & 34 & 1,09 & DEMOCRACIA & 84 & 1,37 \\
\hline ARTE & 33 & 1,06 & ZELAYA & 84 & 1,37 \\
\hline CONTRA & 33 & 1,06 & CUARTA & 79 & 1,29 \\
\hline CRISIS & 33 & 1,06 & PODER & 79 & 1,29 \\
\hline OEA & 31 & 0,99 & JUNIO & 66 & 1,07 \\
\hline ECONOMÍn & 30 & 0,96 & TODOS & 66 & 1,07 \\
\hline ZELAYA & 30 & 0,96 & PAÍl & 64 & 1,04 \\
\hline
\end{tabular}

En posiciones mucho menos importantes aparecen en RDS palabras denotativas del campo que queda invisibilizado en $\mathrm{HNH}$ como: democracia, militares, derechos, libertad, información, resistencia, justicia, golpistas, miedo. Por lo tanto, es necesario tener en cuenta esto en el uso del conteo de palabras mediante programas informáticos, pues las primeras posiciones marcan una tendencia pero, al menos en este estudio, tienden a igualarse, mientras que en las posiciones menos importantes tienden a diferir.

\section{Conclusiones}

El corpus una vez puesto en valor y estudiado se convierte en un lugar de memoria y testimonio del acontecer de la sociedad hondureña. Por la monumentalidad del corpus, son una plataforma documental sólida y de gran diversidad. La heterogeneidad del material viene de la incorporación de varios soportes ${ }^{10}$, pero a través de esta diversidad se percibe la producción de una cultura particular, de cierta ciudad letrada y cibernética.

Si bien puede notarse en los diarios que los titulares sobre la situación política en el país son alarmantes muchas semanas antes del golpe en la revista electrónica de la Red de Desarrollo Sostenible es una semana antes de la fecha fijada para la encuesta -el 28 de junio - que inicia una tormenta de intercambios cuestionando la sociedad y la política hondureñas, como puede verse por los títulos de los envíos.

A pesar de que los programas informáticos proponen una forma sistemática para tratar la información en base a la Teoría Fundamentada, es necesario enriquecer este aporte con otros tipos de análisis, tales como el análisis sociológico del sistema de discursos, el dialogismo de Bajtín, la sociología cultural y la hermenéutica-entre otros; así como otras teorías de las ciencias sociales que permiten interpretar más a fondo los hallazgos. Sin embargo, para la finalidad de este artículo, se considera que la exposición de estos procedimientos pueden ser un aporte para inspirar futuras investigaciones.

Mi objetivo sigue siendo el de contribuir a la construcción de una metodología en base a las representaciones sociales que rigen los sistemas de identificación y de integración social (Randazzo, 2011, 2012 b), pues la actualidad no se cuenta con "ninguna teoría alternativa seria que explique cómo las estructuras sociales, incluyendo las de los contextos comunicativos, pueden restringir las estructuras del texto y la conversación" (van Dijk, 1998). No obstante, en el intento de acceder a lo visible hipotético de la invisibilidad social, es decir esas estructuras que han venido a llamarse imaginarios sociales, sería necesario aventurarse en desvelar los esquemas que subyacen en estas construcciones de la realidad social.

10. Los aportes de RDS son de diversos tipos: comentarios, artículos de opinión de particulares, referencias a páginas electrónicas, poemas, videos, etc. Este tipo de material -a pesar de ser eminentemente lenguaje escrito - presenta cierto grado de oralidad, reforzado por un matiz coloquial, a veces un poco al estilo blog. En cambio los de HNH son titulares de articulos periodísticos. 


\section{Bibliografía}

- Coca, Juan; Valero, Jesús; Randazzo, Francesca; Pintos, Juan Luis (coord.) (2011) Nuevas posibilidades de los imaginarios sociales. Santa Uxía de Riveira: CEASGA.

- Conde, Fernando (2009) Análisis sociológico del sistema de discursos. Madrid: CIS. Cuadernos Metodológicos, 43.

- Castoriadis, Cornelius (1975) L'institution imaginaire de la société. Paris: Éditions du Seuil.

- Charmaz, Kathy (2008) Constructing Grounded Theory. London: Sage.

- Lakoff, George y Rockridge Institute (s.f.) Thinking Points: Communicating Our American Values and Vision. http://www.cognitivepolicyworks.com/wordpress/ wp-content/uploads/Thinking-Points-BETA1.pdf, recuperado el 21 de marzo de 2012.

- Pintos, J. L. (2001), "Construyendo realidad(es): los Imaginarios Sociales", Realidad. Revista del Cono Sur de Psicología Social y Política, 1: 7-24.

- Pintos, Juan Luis (2004) "Inclusión-exclusión. Los imaginarios sociales de un proceso de construcción social", SEMATA, Ciencias Sociais e Humanidades, vol. 16, pp. 17-52.

- Pintos, Juan L. (2005): "Inclusión / Exclusión. Los imaginarios Sociales de un Proceso de construcción social", Revista Sémata, Facultad de Geografía e Historia. No. 16: 1-50.

- Pintos, Juan L. (2006): "Imaginarios y medios de comunicación”. En: Bouzada Fernández, X. (coord.) Cultura e novas tecnoloxías, Consello da Cultura Galega, Santiago de Compostela (España): 21-44.

- Randazzo, Francesca (2011) "Realitats i imaginaris". Ciutadans 8, 01/12, Andorra, pp. 38-46.

- Randazzo, Francesca (2012a) "Golpe de Estado en Honduras, colisión entre lo real y lo imaginario". RIPS, "Investigación social en torno a los imaginarios sociales", Vol.11, No.2, pp. 173-182.

- Randazzo, Francesca (2012b) "Los imaginarios sociales como herramienta", Imagonautas, $\mathrm{N}^{\mathrm{o}} 2$, vol. 2, pp. 7796.

- Randazzo, Francesca (2013) “Aproximaciones al golpe de Estado en Honduras". Realidad, Revista de Ciencias Sociales y Humanidades, 136, abril-junio, pp.231-280.

- Randazzo, Francesca (2015) "Imaginarios de la negación", Revista Inclusiones, Vol.2, No.1, eneromarzo.

- Van Dijk, Teun A. (1998) Ideología. Una aproximación multidisciplinaria. Barcelona: Editorial Gedisa.

- Williams, Raymond (1980) Marxismo y literatura. Barcelona: Ediciones península. 